\title{
Accurate optimization models for interference constrained bandwidth allocation in cellular networks
}

Grit Ecker, Di Yuan, Arie M. C. A. Koster and Anke Schmeink

The self-archived postprint version of this journal article is available at Linköping University Institutional Repository (DiVA):

http:/ / urn.kb.se/ resolve?urn=urn:nbn:se:liu:diva-152795

N.B.: When citing this work, cite the original publication.

Ecker, G., Yuan, Di, Koster, A. M. C. A., Schmeink, A., (2019), Accurate optimization models for interference constrained bandwidth allocation in cellular networks, Computers \& Operations

Research, 101, . https:/ / doi.org/ 10.1016/j.cor.2018.08.011

Original publication available at:

https:/ / doi.org/ 10.1016/j.cor.2018.08.011

Copyright: Elsevier

http:/ / www.elsevier.com/

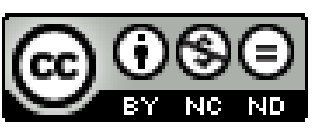




\title{
Accurate Optimization Models for Interference Constrained Bandwidth Allocation in Cellular Networks
}

\author{
Grit Ecker ${ }^{\mathrm{a}}$, Di Yuan ${ }^{\mathrm{b}, \mathrm{c}}$, Arie M. C. A. Koster ${ }^{\mathrm{d}, *}$, Anke Schmeink ${ }^{\mathrm{e}}$ \\ ${ }^{a}$ INFORM GmbH, Risk E Fraud Division, Aachen, Germany \\ ${ }^{b}$ Department of Science and Technology, Linköping University, Norrköping, Sweden \\ ${ }^{c}$ Department of Information Technology, Uppsala University, Uppsala, Sweden \\ ${ }^{d}$ Lehrstuhl II für Mathematik, RWTH Aachen University, Aachen, Germany \\ ${ }^{e}$ Institute for Theoretical Information Technology, RWTH Aachen University, Aachen, Germany
}

\begin{abstract}
In cellular networks, the signal-to-interference-plus-noise ratio (SINR) is a key metric for link availability and quality. For network planning purposes, a straightforward modeling unfortunately yields numerically difficult optimization models. Further, given a required data rate of a link, its bandwidth consumption depends nonlinearly on the SINR.

In this paper, we develop two novel approaches to jointly model SINR-based link availability and bandwidth requirements accurately. The first approach is a set-wise formulation from a user's point of view, while the second one exploits discrete channel quality indicators. We compare these formulations with three known approximate approaches numerically, revealing the clear outperformance of our approaches in terms of exactness. Moreover, since the exact models comprise an exponential number of either variables or constraints, we discuss their pros and cons in a further computational study and develop a more efficient algorithm dealing implicitly with the involved constraints.
\end{abstract} Keywords: network optimization, interference modeling, mixed integer programming.

\footnotetext{
${ }^{*}$ Corresponding author

Email addresses: grit.ecker@rwth-aachen.de (Grit Ecker), diyua@itn.liu.se (Di Yuan), koster@math2.rwth-aachen.de (Arie M. C. A. Koster), schmeink@ti.rwth-aachen.de (Anke Schmeink)
} 


\section{Introduction}

One major challenge for the planning of cellular networks is to manage interference. For instance, so-called inter-cell interference is caused by signals from other cells which use the same frequency for transmission. Thus, a receiver does not only receive the intended signal from the base station (BS) within its cell but also undesired signals from other BSs.

In this paper, we focus on the planning of cellular networks in which inter-cell interference is to be constrained. Whereas in networks of the second generation $(2 \mathrm{G})$, frequency assignment was used [1], from the third generation (3G) on, signal-to-interferenceplus-noise ratio (SINR) conditions have to be satisfied for good signal perception; see, e. g., [2]. Such requirements ensure that the ratio between the signal strength of the desired signal and the total strength of the interfering signals plus some background noise is sufficiently high to establish a physical link from the transmitter to the receiver. The derived physical model is widely used in the literature to limit or prevent interference in various kinds of resource allocation scenarios occurring for different types of wireless communication networks.

With increasing traffic volumes, not only link availability, but also bandwidth requirements have become critical and require an accurate modeling. Here, the bandwidth required for transmission at a requested data rate depends on the SINR as well. Whereas modeling SINR conditions for link availability has received considerable attention in the literature (see below), an accurate and numerically stable modeling of the bandwidth consumption has, to our knowledge, not been proposed.

Contribution and Outline. In this paper, we present two approaches for the integrated modeling of the SINR conditions for both link availability and bandwidth consumption. One formulation is a set-wise model and the other one is based on discrete values for the quality of a link. We compare our novel exact formulations to three known, but approximate approaches regarding bandwidth consumption (and coverage). A computational study reveals the clear superior performance of our new approaches. Additionally, we compare our exact models with each other in terms of number of variables, number of constraints, and computing time. Due to the exponential number of either 
variables or constraints, instances with a higher number of BSs cannot be solved in a straightforward way. Hence, we additionally develop an alternative algorithm based on separation of the exponentially many constraints. A further numerical evaluation reveals the good potential of this approach. The contributions we present are based on investigations presented in the first author's $\mathrm{PhD}$ thesis [3].

The remainder of this paper is organized as follows. A survey on SINR modeling closes this section. In Section 2, we depict a first complete, but nonlinear, formulation for the planning of a cellular wireless network together with the necessary notation. Subsequently in Section 3, we review and adapt three known linear formulations that model SINR-based link availability exactly or approximately. They are combined with a relaxed version of the capacity constraints. Next, in Section 4 we develop two novel distinct exact formulations. In Section 5, we discuss the pros and cons of the exact formulations in a numerical comparison. Additionally, we present a computational comparison between the various (in)accurate formulations. We conclude with some final remarks in Section 6 .

Related work. For a general introduction to wireless network design, including the classical way of formulating SINR constraint, we refer to [4]. For a more recent collection of studies on various topics of optimizing wireless systems, see [5]. One line of research has been resource allocation in ad hoc and mesh networks. Starting with [6], the problem of scheduling wireless links respecting the SINR model has attracted algorithmic-oriented research, focusing on approximation algorithms for link scheduling and power control. For example, for arbitrary wireless networks with variable transmission powers, the number of connections satisfying SINR constraints is maximized in [7]. Recently, the authors in [8] investigate two basic approaches of modeling SINR conditions for parallel link transmissions in wireless networks, and introduce matching inequalities to improve the optimality gaps. In [9], wireless mesh networks where data is distributed in the network cooperatively by all nodes is studied. The authors use SINR conditions to determine interfering links for every node as to assign channels, distribute capacity and determine link flows.

In [10], the authors presented a complex mixed integer programming model for 
the planning of cellular wireless networks taking, among other aspects, signal quality constraints into account. The linearization of the SINR condition leads to numerical difficulties in handling these constraints, cf. [11]. The authors in [12] propose simple models and add the complexity step-by-step for cellular network planning with conditions on the SINR. The model addressing SINR becomes rather complex and is solved using a tabu search heuristic. Genetic algorithms have been proposed in [13], to solve a wireless network planning problem with power and frequency assignment subject to SINR consideration. In [14], the authors discuss recent advances in solving integer programs for addressing the numerical instabilities originating from SINR constraints. The issue of numerically difficult SINR constraints is also present in other types of networks, such as terrestrial broadcasting [15] where emission powers can be adapted to obtain a good signal-to-interference-plus-noise ratio. In [16], an alternative, numerically stable SINR-model is presented, see Section 3 In [17], a subset of the inequalities of [16] that is relatively easy to separate, is considered. Moreover, in [18], the authors apply power discretization in wireless network design problems and replace SINR constraints by bounds on sums of binary variables. The authors show numerically that their approach outperforms earlier formulations (like [10]) that suffer from numerical instabilities. More details of the theoretical aspects of [18], along with a hierarchical classification of wireless network design problems, are provided in a $\mathrm{PhD}$ thesis [19] (see also [20]). Note that for given BS deployment and association between users and BSs, the SINR condition is easy to check and forms a linear equation system if power control is present [21]. We also remark that SINR-type of constraints remain of high relevance to new, more advanced transmission technique, such as interference cancellation [22].

Apart from heuristics and deriving strong integer programming formulations, Benders decomposition has been considered for wireless network optimization. In [23], Benders cut is used in a branch-and-bound framework for power minimization. Interference is however not part of the consideration. In [24], the authors consider a cellular network planning problem that is similar to ours, and apply combinatorial Benders decomposition, where the subproblem contains some of the integer variables, for problem solution. 
In addition to modeling SINR, other important aspects in performance engineering of wireless networks include multi-objective optimization and solution robustness. For developments related to these two aspects, we refer to [25, 26, 27] and [28, 29, 30, 31, 32], respectively. The works are of significance, in particular because some of them include SINR considerations.

Among the aforementioned references, [16, 18, 24] are closely related to the current work. The basic idea of cover-type inequalities in Section 4.2 has been developed in [16, 18], both having the motivation that the conventional way of modeling SINR using big-M leads to very poor numerical stability. The work in [16] considers link activation in generic wireless networks with one single SINR level. This problem setup lacks some of the key elements for cellular network planning, notably assignment of users to base stations, which is addressed in the current paper. In comparison to [18], the model in Section 4.2 has clear similarities, including that [18] uses (generalized upper bound) cover inequalities with discrete power levels. The differences consist in the problem setup, as we consider both cost and service coverage as objectives along with multiple service quality levels, and the solution procedure in which different sets of inequalities are separated exactly in our case. Reference [24] considers a problem quite similar to ours, and proposes the use of Benders decomposition. The idea of this approach is similar to ours, in the sense that it attempts to solve a small-size integer program while generating cuts repeatedly. However, the type of cuts is different. Moreover, the Benders cuts in [24] still use big-M coefficients, which are no longer present in our formulation. Finally, a significant difference of our work to [16, 18, 24] is the derivation of the user-oriented model in Section 4.1. which is not present in any of the references.

We end the section by highlighting the practical relevance of the problem that we study. First, SINR is a key performance indicator of wireless networking, as demonstrated by the references mentioned above addressing optimization considering SINR. For the current fourth generation $(4 \mathrm{G})$ cellular networks, all BSs reuse the same frequency spectrum, representing the interference scenario we consider, and the same radio transmission technology is expected to be used also for the upcoming fifth generation $(5 \mathrm{G})$ networks, Moreover, the tranmission power is usually fixed in $4 \mathrm{G}$, whereas 
a number of coding and modulation schemes, each corresponding to a minimum SINR level, have been defined. These aspects are part of our problem definition. Thus far, engineering practice tends to use various types of approximations (e.g., the so called conflict-graph model) to address interference, mainly due to the difficulty of incorporating mathematically SINR constraints. Hence demonstrainting the viability of accurately and effectively modeling SINR for problem solving is of significance for current network planning methods and tools (e.g., [33, 34]). To this end, we believe our study represents a step towards pursuing the potential of optimization for the target application of cellular network deployment.

\section{Problem Description}

In this section, we introduce the notation and present a first complete formulation to optimize the location and configuration of BSs in a cellular access network. Capacity and interference are modelled by nonlinear constraints which are linearized in the following sections. The proposed formulation is inspired by [35] and its extension addressing robust optimization is developed in [36]. The cellular network we consider in this paper consists of BSs and mobile users. The location and all other configuration parameters of a BS are consolidated in a BS candidate site $s \in \mathcal{S}$ with $\mathcal{S}$ denoting the set of all BS candidate sites. Note that multiple candidate sites $s \in \mathcal{S}$ can have the same geographical location, but differ in, for example, the transmission power, azimuth, or tilt. For simplicity, we speak of BSs instead of BS candidate sites henceforth. Each BS has cost $c_{s}$ and provides a total downlink bandwidth $b_{s}$ which constitutes the capacity of the BS in $\mathrm{Hz}$.

A mobile user has to be assigned to at most one BS (call admission) and has to be allocated the requested bit rate. To reduce the number of users which have to be taken into account in the optimization models and to account for movements and fluctuations in demands, we merge demands of users in a small area to a single traffic demand node (TN) based on the concept presented in [37]. We then denote the set of TNs by $\mathcal{T}$ and each $\mathrm{TN} t \in \mathcal{T}$ requests a data rate $w_{t}$ (in bps).

The Cellular Network Planning Problem (CNPP) is in essence a capacitated facility 
location problem. We have to decide which BS candidates to deploy and to assign TNs to BSs such that bandwidth capacities are not exceeded. In contrast to the wellknown capacitated facility location problem, the bandwidth capacity consumed by a $\mathrm{TN}$ depends on the BS it is assigned to and on all other BSs deployed in the vicinity of the TN. In fact, it is possible that it cannot be assigned to any of the BSs in its vicinity without consuming more bandwidth than available. Therefore, not every TN has to be assigned to a BS, and the CNPP objective is twofold: on the one hand to minimize the deployment cost and on the other hand to maximize the number of TNs covered. Hence, the CNPP is a multi-criteria optimization problem with contradicting objective functions. In this work, these two objectives are combined in a single objective by a scaling factor, see (3a) below.

Before we can present a first integer programming (IP) formulation, the bandwidth consumption of an assignment has to be specified: Modern cellular networks utilize orthogonal transmission schemes such as the Orthogonal Frequency Division Multiple Access (OFDMA) modulation scheme. One of the advantages is that intra-cell interference can be neglected. To determine inter-cell interference at $\mathrm{TN} t$ assigned to BS $s$, the signal-to-interference-plus-noise (SINR) function $\gamma_{s t}(x)$ as a function of the binary vector $x \in\{0,1\}^{|\mathcal{S}|}$ denoting the installed BSs have to be computed:

$$
\gamma_{s t}(x):=\frac{P_{\mathrm{r}}(s, t)}{\sum_{\sigma \in \mathcal{S}_{s t}(x)} P_{\mathrm{r}}(\sigma, t)+\eta},
$$

where $P_{\mathrm{r}}(s, t)$ denotes the received power at TN $t$ from BS $s, \eta$ denoting the background noise, and $\mathcal{S}_{s t}(x)$ the set of installed BSs interfering the signal from $s$ to $t$ (a BS is interfering if the received power is above a certain threshold). The received power $P_{\mathrm{r}}(s, t)$ at TN $t$ from BS $s$ is computed as $P_{\mathrm{r}}(s, t)=p_{s} a_{s t}$ with $p_{s}$ denoting the transmission power of $s$ and $a_{s t}$ the total power gain between $s$ and $t$. The unit of the SINR given by (1) is watt (W). The SINR in $\mathrm{dB}$ is computed as

$$
\gamma_{s t}^{\mathrm{dB}}=10 \cdot \log _{10}\left(\gamma_{s t}\right) .
$$

To guarantee a certain link quality, we introduce a function $e_{s t}(x)$ denoting the spectral efficiency for the link from BS $s$ to TN $t$. This function is exactly calculated as

$$
e_{s t}(x)=\log _{2}\left(1+\gamma_{s t}(x)\right) .
$$


To establish a transmission link, the spectral efficiency must exceed a threshold $e_{\min }>$ 0 . Therefore, $e_{s t}(x)$ is set to zero, if the value calculated by (2) falls below $e_{\min }$.

To avoid unnecessary decision variables for impossible assignments, we consider the spectral efficiency in case no further interfering BSs are deployed, i.e., on basis of the signal-to-noise ratio $P_{r}(s, t) / \eta$. In this way, an upper bound $\bar{e}_{s t}$ on the real spectral efficiency $e_{s t}$ is computed and the following auxiliary sets of indices are defined:

$$
\begin{aligned}
\mathcal{S} * \mathcal{T} & :=\left\{(s, t) \in \mathcal{S} \times \mathcal{T} \mid \bar{e}_{s t} \geq e_{\min }\right\}, & \\
\mathcal{S}_{t} & :=\{s \in \mathcal{S} \mid(s, t) \in \mathcal{S} * \mathcal{T}\} & \forall t \in \mathcal{T}, \\
\mathcal{T}_{s} & :=\{t \in \mathcal{T} \mid(s, t) \in \mathcal{S} * \mathcal{T}\} & \forall s \in \mathcal{S} .
\end{aligned}
$$

The set $\mathcal{S} * \mathcal{T}$ consists of all BS-TN pairs for which the establishment of a link is potentially possible. Based on this set, $\mathcal{S}_{t}$ is the set of all BSs which can provide the minimum required spectral efficiency to $\mathrm{TN} t$. Similarly, $\mathcal{T}_{s}$ denotes the set of TNs for which a link to BS $s$ has sufficient spectral efficiency.

Let $x_{s} \in\{0,1\}$ indicate whether BS $s \in \mathcal{S}$ is deployed and $z_{s t} \in\{0,1\}$ whether TN $t$ is assigned to BS $s$ with $(s, t) \in \mathcal{S} * \mathcal{T}$. Furthermore, for simplicity of the objective function we introduce an auxiliary variable $u_{t}$ which is equal to one if TN $t$ is not served by any BS.

We formalize the basic formulation of the CNPP in the following integer (nonlinear) program.

$$
\begin{aligned}
& \min \sum_{s \in \mathcal{S}} c_{s} x_{s}+\lambda \sum_{t \in \mathcal{T}} u_{t} \\
& \text { s.t. } \sum_{s \in \mathcal{S}_{t}} z_{s t}+u_{t}=1 \quad \forall t \in \mathcal{T} \\
& \sum_{t \in \mathcal{T}_{s}} \frac{w_{t}}{e_{s t}(x)} z_{s t} \leq b_{s} x_{s} \quad \forall s \in \mathcal{S} \\
& z_{s t} \leq x_{s} \quad \forall(s, t) \in \mathcal{S} * \mathcal{T} \\
& x_{s}, z_{s t}, u_{t} \in\{0,1\}
\end{aligned}
$$

The objective function (3a) minimizes the number of deployed BSs while the number of served TNs is maximized. The two objecting functions are combined by the scaling 
Table 1: Spectral efficieny based on S(I)NR requirements (in $\mathrm{dB}$ ) for LTE and $10 \mathrm{MHz}$ bandwidth according to $[38]$.

\begin{tabular}{l|cccccccccccccccccc}
\hline CQI & 0 & 1 & 2 & 3 & 4 & 5 & 6 & 7 & 8 & 9 & 10 & 11 & 12 & 13 & 14 & 15 \\
\hline start [ & $-\infty$ & -5.1 & -2.9 & -1.7 & -1 & 2 & 4.3 & 5.5 & 6.2 & 7.9 & 11.3 & 12.2 & 12.8 & 15.3 & 17.5 & 18.6 \\
end ) & -5.1 & -2.9 & -1.7 & -1 & 2 & 4.3 & 5.5 & 6.2 & 7.9 & 11.3 & 12.2 & 12.8 & 15.3 & 17.5 & 18.6 & $\infty$ \\
spec. eff. & - & 0.25 & 0.4 & 0.5 & 0.66 & 1 & 1.33 & 1.5 & 1.6 & 2 & 2.66 & 3 & 3.2 & 4 & 4.5 & 4.8 \\
\hline
\end{tabular}

parameter $\lambda>0$. The connection between variables $z$ and $u$ is formalized in the coverage constraints (3b). Furthermore, constraints (3c) represent the capacity constraints, which ensure that the bandwidth allocated to the TNs served by a single BS does not exceed its capacity. Note that for a fixed $x$, these constraints are knapsack constraints (and hence, the problem is NP-hard). Finally, constraints (3d) denote variable upper bound constraints which represent auxiliary inequalities and guarantee that a TN can only be assigned to a BS which is installed. In total, IP (3) comprises both $|\mathcal{S}|+|\mathcal{T}|+|\mathcal{S} * \mathcal{T}|$ variables and constraints.

The major disadvantage of formulation (3) is clearly the nonlinearity of the capacity constraints (3c). They not only model (to our knowledge for the first time) accurately the capacity consumption, but implicitly also the SINR requirements needed in cellular networks. That is, if the SINR $\gamma_{s t}(x)$ drops below a threshold, no transmission link between $s$ and $t$ can be established. In such cases, the function $e_{s t}(x)$ simply returns zero, and the bandwidth consumption of this assignment would be infinite, implying variable $z_{s t}$ is forced to zero.

The values of the spectral efficiency computed according to (2) are numerical difficult, not only in optimization, but also in practice. Therefore, the spectral efficiency function is usually approximated by a stepwise constant function. For this, we use the look-up Table 1 taken from [38], which maps a range of SINR values (including interference) or SNR values (no interference) given in $\mathrm{dB}$ to one discrete spectral efficiency for a bandwidth of $10 \mathrm{MHz}$. For example, the interval $[-5.1,-2.9)$ is associated with the spectral efficiency 0.25 . Thus, the second line in Table 1 denotes the left boundary of the SINR interval, the third line denotes the right boundary, and the fourth line denotes the associated spectral efficiency. Moreover, each line is labeled by a channel 
quality indicator (CQI) in the first line.

Although this stepwise function simplifies computations, model (3) remains computationally intractable. For a computational more tractable model, we have to linearize the capacity constraints $3 \mathrm{c}$, taking into account both aspects, the SINR and the bandwidth consumption. In Section 3, we first review and adapt existing linearizations for the SINR requirement, approximating the bandwidth consumption in a rudementary fashion. Next, in Section 4 two novel formulations for the integrated modelling of capacity and SINR requirements are presented.

\section{Modelling SINR Requirements}

The two most commonly used interference models are (i) graph-based and (ii) fading channel or physical models [2]. A physical model that is accurate and numerically stable is discussed first, while we adapt two graph-based models in Sections 3.2 and 3.3 .

\subsection{SINR Constraints via Cover Inequalities}

A common way to formulate the SINR requirement is

$$
\gamma_{s t}(x) \geq \delta,
$$

stating that the ratio should be above a predefined threshold $\delta$. Recall the definition of $\gamma_{s t}(x)$ is given by (11). For notational convenience, let $\mathcal{S}_{s t} \subseteq \mathcal{S}_{t} \backslash\{s\}$ denote the set of potentially interfering BSs.

To include the SINR requirement (4) in the basic optimization problem (3), we have to reformulate it as a linear inequality. To this end following [12], we add the following big- $M$ constraints, exploiting the decision variables $z_{s t}$ and $x_{\sigma}$ :

$$
P_{\mathrm{r}}(s, t) z_{s t}+M_{s t}\left(1-z_{s t}\right) \geq \delta\left(\sum_{\sigma \in \mathcal{S}_{s t}} P_{\mathrm{r}}(\sigma, t) x_{\sigma}+\eta\right) \quad \forall(s, t) \in \mathcal{S} * \mathcal{T},
$$

with $M_{s t}$ being sufficiently large, e. g., $M_{s t}:=\delta\left(\sum_{\sigma \in \mathcal{S}_{s t}} P_{\mathrm{r}}(\sigma, t)+\eta\right)$ making the constraint redundant whenever $z_{s t}=0$.

Solving models with big- $M$ constraints is in general numerically difficult due to the weak LP relaxation and the precision of LP solvers. But handling constraints (5) 
is numerically even more difficult since the received powers $P_{\mathrm{r}}(s, t)$ and $P_{\mathrm{r}}(\sigma, t)$ can vary significantly in magnitude [11]. The authors of [16] derive cover inequalities to replace the conventional SINR constraints (5) and to overcome numerical instabilities as described in the following. Since the big- $M$ constraint $[5$ for one $(s, t) \in \mathcal{S} * \mathcal{T}$ is only restrictive if $\mathrm{TN} t$ is assigned to $\mathrm{BS} s$, it reduces to

$$
P_{\mathrm{r}}(s, t) \geq \delta\left(\sum_{\sigma \in \mathcal{S}_{s t}} P_{\mathrm{r}}(\sigma, t) x_{\sigma}+\eta\right)
$$

if $z_{s t}=1$. Defining

$$
r_{s t}:=\frac{P_{\mathrm{r}}(s, t)}{\delta}-\eta
$$

constraint 6 is equivalent to

$$
\sum_{\sigma \in \mathcal{S}_{s t}} P_{\mathrm{r}}(\sigma, t) x_{\sigma} \leq r_{s t}
$$

which is a knapsack constraint. Knapsack constraints can be alternatively formulated by the set of all cover inequalities (i.e., a binary vector is feasible if and only if it satisfies all cover inequalities [39]. In the present context, a subset $\mathcal{C}_{s t} \subseteq \mathcal{S}_{s t}$ is a cover if

$$
\sum_{\sigma \in C_{s t}} P_{\mathrm{r}}(\sigma, t)>r_{s t}
$$

with corresponding cover inequality

$$
\sum_{\sigma \in C_{s t}} x_{\sigma} \leq\left|C_{s t}\right|-1
$$

However, this inequality should only be restrictive if $\mathrm{TN} t$ is assigned to BS $s$, i. e., if $z_{s t}=1$. Hence, we exchange the 1 of the right hand side with the assignment variable and obtain the cover inequality

$$
\sum_{\sigma \in C_{s t}} x_{\sigma} \leq\left|\mathcal{C}_{s t}\right|-z_{s t}
$$

Hence, SINR constraints (5) can be replaced by all cover inequalities (8) for all pairs $(s, t) \in \mathcal{S} * \mathcal{T}$. For each $\mathrm{TN} t$, there exist at most $\left|\mathcal{S}_{t}\right|$ possible serving BSs and thus, at 
$\operatorname{most}\left|\mathcal{S}_{t}\right| \cdot 2^{\left|\mathcal{S}_{t}\right|-1}$ covers. In total, at most $\sum_{t \in \mathcal{T}}\left|\mathcal{S}_{t}\right| \cdot 2^{\left|\mathcal{S}_{t}\right|-1}$ many cover inequalities $(8)$ have to be added to the basic formulation (3) to obtain a complete formulation.

To avoid this exponential number of constraints, as in [16], we separate only inequalities violated by an integer feasible solution as follows (exploiting the technique of delayed row generation [39]). Let $(\tilde{x}, \tilde{z}, \tilde{u})$ be an integer solution of (3). For every $t \in \mathcal{T}$, we define the serving BS $s \in \mathcal{S}_{t}$ with $\tilde{z}_{s t}=1$ and the set of interfering BSs $\mathcal{S}_{t}^{\prime}:=\left\{\sigma \in \mathcal{S}_{t} \backslash\{s\} \mid \tilde{x}_{s}=1\right\}$. If

$$
\frac{P_{\mathrm{r}}(s, t)}{\sum_{\sigma \in \mathcal{S}_{t}^{\prime}} P_{\mathrm{r}}(\sigma, t)+\eta}<\delta,
$$

we add a cover inequality (8) with $C_{s t}:=\mathcal{S}_{t}^{\prime}$. The violation of this inequality is 1 for solution $(\tilde{x}, \tilde{z}, \tilde{u})$. By greedily removing elements from the $\operatorname{set} C_{s t}$, a minimal cover can be determined, resulting in stronger inequalities.

If the bandwidth consumption in the capacity constraints $3 \mathrm{c}$ is approximated by its best possible value $w_{t} / \bar{e}_{s t}$ (the case where only noise interfers with the signal), we denote the formulation (3) together with the separation of cover inequalities (8) described in this paragraph by SINR cover formulation and use the acronym SCF. It guarantees that a link from a BS to a TN is established if and only if the associated SINR is sufficiently large. Hence, no violated SINR condition occurs for an optimal solution. However, we cannot guarantee that the capacity constraints $3 \mathrm{Bc}$ are de facto satisfied as the spectral efficiencies are based on the SNR, and hence the solution may violate the capacity limit for SINR.

\subsection{Conflict Graph}

In the context of wireless networks, the concept of a conflict graph has been widely used to describe pairs of BSs that cannot be operated with the same configuration simultaneously (e.g., in $2 \mathrm{G}$ networks, these BSs could not be assigned the same frequency [1]). In our setting, a conflict graph $G=(\mathcal{S}, \mathcal{E})$ can be used to limit inter-cell interference and to avoid explicit SINR constraints in the model, see also [40]. Only non-adjacent BSs can be exploited simultaneously, i.e., the selection forms an independent set in the graph. Assuming a single power emission level for all BSs, a reasonable 
definition of the edge set $\mathcal{E}$ is a minimum distance requirement, i. e., two BSs are adjacent if the distance between them is less than a minimum required distance $d_{\min }$. Concerning macro cells, $d_{\min }=500 \mathrm{~m}$ is a common value [41]. An independent set in a graph is a subset $\mathcal{S}^{\prime} \subseteq \mathcal{S}$ of the vertices such that there does not exist an edge $i j \in \mathcal{E}$ for all $i, j \in \mathcal{S}^{\prime}$. This restriction is reflected in the constraints

$$
x_{i}+x_{j} \leq 1 \quad \forall i j \in \mathcal{E} \text {. }
$$

The concept of conflict or interference graphs is a commonly applied technique; see, e. g., [40]. It has been employed in the planning of global system for mobile communications (GSM) networks [42], wireless local area networks (WLANs) [43], and LTE networks [44], and in a modified way via complement sets for the deployment of cooperation clusters in general wireless cellular networks [45] to name just some works.

By taking the convex hull of all independent sets we arrive at the independent set polytope, for which, however, a complete description is unknown. Instead, two alternative linear formulations exist (resulting in polytopes containing the independent set polytope). The first one consists of constraints (9) and nonnegativity of the variables and is known as the edge formulation. A stronger formulation is obtained by replacing constraints (9) by maximal clique inequalities [46], where a clique is a complete subgraph (the clique formulation). Formally speaking, let $\mathcal{U} \subseteq \mathcal{S}$ be a subset of the vertex set. We call $\mathcal{U}$ a clique of conflict graph $G=(\mathcal{S}, \mathcal{E})$ if there exists an edge $i j \in \mathcal{E}$ for all $i, j \in \mathcal{U}$. A clique is maximal if it is not included in a larger clique. The convex hull of binary solutions satisfying all maximal clique inequalities

$$
\sum_{s \in \mathcal{U}} x_{s} \leq 1 \quad \forall \mathcal{U} \subseteq \mathcal{S}, \mathcal{U} \text { max. clique in } G
$$

is identical to the independent set polytope, but its LP relaxation is more restrictive than the LP relaxation of the edge formulation.

The problem of finding a clique of maximum cardinality in a graph with $n$ vertices is NP-hard [47]. Hence, it is unlikely to enumerate all maximal cliques in polynomial time. All maximal cliques can be computed by, e.g., the Bron-Kerbosch algorithm [48] with complexity $O\left(3^{n / 3}\right)$ [49] which is fast enough for our purposes. For larger in- 
stances, more advanced algorithms for maximal clique enumeration are available (e.g., [50]), or inequalities (10) might be separated on the fly.

By (again) replacing the spectral efficiency $e_{s t}(x)$ in $3 \mathrm{c}$ by its best possible value $\bar{e}_{s t}$ and incorporating the maximal clique inequalities [10], we obtain the conflict graph formulation and use the acronym CGF for it. This model can only limit the inter-cell interference but may violate both SINR conditions (4) and capacity constraints (3c).

\subsection{TN Coverage Requirement}

The model which performed best among the approximate formulations discussed in [3] is based on the work [51]. It demands that a TN $t$ can be covered by a BS $s$ if the ratio between the SNR-based spectral efficiencies of the serving BS and any interfering BS $\sigma$ exceeds a threshold $\delta_{\mathrm{c}}$, which is related to SINR thresholds. This is modeled by the TN coverage requirement

$$
\frac{\bar{e}_{s t}}{\bar{e}_{\sigma t}} \geq \delta_{\mathrm{c}} \quad \forall \sigma \in \mathcal{S}_{s t} .
$$

Note that $\bar{e}_{\sigma t} \geq e_{\min }>0$ per definition of $\mathcal{S}_{t}$, which is the superset of $\mathcal{S}_{s t}$. We formulate the linear model constraints as follows.

$$
z_{s t}+x_{\sigma} \leq 1 \quad \forall t \in \mathcal{T}, s \in \mathcal{S}_{t}, \sigma \in \mathcal{S}_{s t} \text { with } \frac{\bar{e}_{s t}}{\bar{e}_{\sigma t}}<\delta_{\mathrm{c}}
$$

which also represent a type of a conflict graph with $(s, t)$ and $\sigma$ forming an edge if $\frac{\bar{e}_{s t}}{\bar{e}_{\sigma t}}<\delta_{\mathrm{c}}$. This graph is however bipartite and thus the maximal clique size is two. For each TN $t$, there exist $\left|\mathcal{S}_{t}\right|$ potential serving BSs and at most $\left|\mathcal{S}_{t}\right|-1$ interfering BSs

for an $(s, t)$-pair. Hence, we add at most $\sum_{t \in \mathcal{T}}\left|\mathcal{S}_{t}\right| \cdot\left(\left|\mathcal{S}_{t}\right|-1\right)=\sum_{t \in \mathcal{T}}\left|\mathcal{S}_{t}\right|^{2}-\left|\mathcal{S}_{t}\right|$ many constraints (11) to the basic model (3) resulting in the TN coverage requirement formulation (with replacing $e_{s t}(x)$ by $\bar{e}_{s t}$ in (3c) one more time). To denote this formulation, we use the acronym TCRF.

The TN coverage requirement can only guarantee a certain link quality but has no influence on the actual transmission rate. Thus, violations of SINR requirements (4) may occur and also violated capacity constraints 3 (3c) might exist. 


\section{Modelling Capacity Constraints}

In this section, we present two approaches which model both the SINR requirements (4) and the capacity constraints (3C) exactly (a third exact approach can be found in [3], but is left out here due to its underperformance). Both approaches replace the spectral efficiency function $e_{s t}(x)$ by values from Table 1. As these spectral efficiency values cannot be used arbitrarely, additional variables and constraints are derived to guarantee that only feasible configurations are considered.

\subsection{A TN Oriented Formulation}

First, we propose a TN oriented model where interference is defined set-wise on a TN basis. For every TN $t \in \mathcal{T}$, we define $K_{t}$ different configurations where a configuration $k \in \mathcal{K}_{t}:=\left\{1, \ldots, K_{t}\right\}$ comprehends a set $\mathcal{S}^{k} \subseteq \mathcal{S}_{t}$ of deployed BSs (and all other BSs in $\mathcal{S}_{t} \backslash \mathcal{S}^{k}$ are not deployed) and an accentuated BS $s^{k} \in \mathcal{S}^{k}$ which is the serving BS for $t$. Furthermore, we define configuration $k=0$ so that $\mathrm{TN} t$ is not served by any BS but we do not make any assumption on the deployment of BSs.

For each covered TN $t$ and configuration $k$, we can compute the SINR value $\gamma_{t}^{k}$ as follows.

$$
\gamma_{t}^{k}:=\frac{P_{\mathrm{r}}\left(s^{k}, t\right)}{\sum_{s \in \mathcal{S}^{k} \backslash\left\{s^{k}\right\}} P_{\mathrm{r}}(s, t)+\eta} \quad \forall t \in \mathcal{T}, k \in \mathcal{K}_{t}
$$

Based on these SINR values, we define the associated spectral efficiencies $e_{t}^{k}$ according to Table 1 Note that we consider only configurations with a corresponding spectral efficiency $e_{t}^{k} \geq e_{\min }$.

Finally, we introduce indicator variables $y_{t}^{k}$ which are set to 1 if configuration $k$ is chosen for TN $t$ and 0 otherwise. In case $y_{t}^{k}=1$ for $k \geq 1$, the set $\mathcal{S}^{k}$ of BSs is deployed, all BSs in $\mathcal{S}_{t} \backslash \mathcal{S}^{k}$ are not installed and $s^{k}$ is the serving BS for $t$. If $y_{t}^{0}=1, \mathrm{TN} t$ is not served and we do not assume anything on the deployment of BSs in $\mathcal{S}_{t}$.

We propose the TN oriented formulation denoted by the acronym TOF as the fol- 
lowing ILP.

$$
\begin{aligned}
& \min \sum_{s \in \mathcal{S}} c_{s} x_{s}+\lambda \sum_{t \in \mathcal{T}} y_{t}^{0} \\
& \text { s.t. } \sum_{k=0}^{K_{t}} y_{t}^{k}=1 \quad \forall t \in \mathcal{T} \\
& \sum_{k \in \mathcal{K}^{\prime}: \mathcal{S}^{k}} y_{t}^{k} \leq x_{s} \quad \forall(s, t) \in \mathcal{S} * \mathcal{T} \\
& \sum_{k \in \mathcal{K}_{t}: s \in \mathcal{S}^{k}} y_{t}^{k} \geq x_{s}-y_{t}^{0} \quad \forall(s, t) \in \mathcal{S} * \mathcal{T} \\
& \sum_{t \in \mathcal{T}_{s}} \sum_{k \in \mathcal{K}_{t}: s=s^{k}} \frac{w_{t}}{e_{t}^{k}} y_{t}^{k} \leq b_{s} x_{s} \quad \forall s \in \mathcal{S} \\
& x_{s}, y_{t}^{k} \in\{0,1\}
\end{aligned}
$$

The objective (12a) is the reformulated objective function $3 \mathrm{a}$ in terms of the new variables $y_{t}^{k}$. Constraints (12b) ensure that exactly one configuration is chosen for every TN while $112 \mathrm{c}$ and $\left(12 \mathrm{~d}\right.$ ) connect variables $y_{t}^{k}$ to the BS decision variables $x_{s}$. Note, constraints (12c) are stronger than constraints of the form $y_{t}^{k} \leq x_{s}$, which are comparable to the variable upper bound constraints [3d], as one $x_{s}$ variable simultaneously limits several $y_{t}^{k}$ variables. Finally, the refinement of the capacity constraints $3 \mathrm{c}$ ) is given by $12 \mathrm{e}$. Since it is not immediately clear from the formulation that the configurations selected for different TNs are consistent, we show that this formulation is correct in the following lemma.

Lemma 1. The TOF (12) models the CNPP regarding interference via SINR conditions correctly.

Proof. First of all, the SINR is modelled implicitly in the model by the definition of $\gamma_{t}^{k}$ and $e_{t}^{k}$. Moreover, by the definition of variables $y_{t}^{k}$, parameters $e_{t}^{k}$, and capacity constraints 12e, it is clear that the capacity of an installed BS $s \in \mathcal{S}$ is not exceeded by the assigned TNs. Therefore, to prove correctness of the formulation, it remains to show that the selected configurations are in line with the selected BSs.

By $(12 \mathrm{~b})$, exactly one $y_{t}^{k}$ has to be set to one for each TN $t \in \mathcal{T}$. If $y_{t}^{0}=1,12 \mathrm{c}$ ) and (12d) are trivially satisfied, and the TN does not imply further restrictions. If $y_{t}^{0}=0$, 
(12c) and (12d) reduce to an equality constraint for all $s \in \mathcal{S}_{t}$. For non-installed BS (i.e., $x_{s}=0$ ), all configurations $k \in \mathcal{K}_{t}$ with $s \in \mathcal{S}^{k}$ are set to zero. For every installed BS $s \in \mathcal{S}_{t}$, exactly one configuration from $\left\{k \in \mathcal{K}_{t}: s \in \mathcal{S}^{k}\right\}$ has to be selected. By $(12 \mathrm{~b})$, the selected configuration must belong to

$$
\bigcap_{s \in \mathcal{S}_{t}: x_{s}=1}\left\{k \in \mathcal{K}_{t}: s \in \mathcal{S}^{k}\right\}=\left\{k \in \mathcal{K}_{t}: \mathcal{S}^{k}=\left\{s \in \mathcal{S}_{t}: x_{s}=1\right\}\right\} .
$$

Hence, these configurations only differ by their designated BS $s$ serving TN $t$.

To make the above formulation exact, we needed a (potentially) exponential number of configurations per TN and hence, the ILP can become huge. In fact, ILP (12) comprises $\sum_{t \in \mathcal{T}}\left(K_{t}+1\right)+|S|$ variables and $|\mathcal{S}|+|\mathcal{T}|+2|\mathcal{S} * \mathcal{T}|$ constraints with

$$
K_{t} \leq \sum_{i=1}^{\left|\mathcal{S}_{t}\right|}\left(\begin{array}{c}
\left|\mathcal{S}_{t}\right| \\
i
\end{array}\right) \cdot i \leq\left|\mathcal{S}_{t}\right| \cdot 2^{\left|\mathcal{S}_{t}\right|-1} \quad \forall t \in \mathcal{T} .
$$

Next, we present an alternative exact formulation which is not exponential in the number of variables.

\subsection{Exploiting Discrete Channel Quality Indicators}

In our second model, we exploit the discretization of the spectral efficiencies more directly. In total, we have to consider 15 different values for spectral efficiencies each labeled by the corresponding CQI $k$, which serves as an index. For CQI 0 , no link can be established which is why we do not consider this CQI henceforth. We denote the highest possible CQI for a link from $s$ to $t$, which is associated to the SNR value (no interference), by $\kappa_{s t}$ and the value of the spectral efficiency for any CQI $k$ by $e^{k}$.

To incorporate these discrete CQIs in the basic formulation (3), we introduce new binary variables $z_{s t}^{k}$ for every $(s, t) \in \mathcal{S} * \mathcal{T}$ and every CQI $k \in\left\{1, \ldots, \kappa_{s t}\right\}$. It holds $z_{s t}^{k}=$ 1 if the signal from $s$ to $t$ is established and has the quality of CQI index $k$, thus spectral efficiency $e^{k}$. If TN $t$ is assigned to BS $s$, then this link has exactly one specified CQI. Hence, we can replace the former assignment variables $z_{s t}$ by $\sum_{k=1}^{K_{s t}} z_{s t}^{k}$ for every pair $(s, t) \in \mathcal{S} * \mathcal{T}$. 
The quality of a link is impaired by interfering BSs. If a subset $C \subseteq \mathcal{S}_{t} \backslash\{s\}$ of BSs is deployed, the SINR $\gamma_{s t}(C)$ of the signal from $s \in \mathcal{S}_{t} \backslash C$ to $t$ is calculated as:

$$
\gamma_{s t}(C):=\frac{P_{\mathrm{r}}(s, t)}{\sum_{\sigma \in C} P_{\mathrm{r}}(\sigma, t)+\eta} .
$$

We denote the corresponding CQI by $\kappa_{s t}(C)$. In case that all BSs in $C$ are deployed, better spectral efficiencies than $e^{\kappa_{s t}(C)}$ cannot occur for this link. Hence, $\sum_{k=\kappa_{s t}(C)+1}^{K_{s t}} z_{s t}^{k}=$ 0 . This condition is formulated in the following model constraints.

$$
\sum_{k=\kappa_{s t}(\mathcal{C})+1}^{\kappa_{s t}} z_{s t}^{k} \leq|C|-\sum_{\sigma \in \mathcal{C}} x_{\sigma} .
$$

By observing that the right hand side does not depend on $s$, and $t$ can be assigned to at most one BS, we can strengthen this inequality to:

$$
\sum_{s \in \mathcal{S}_{t} \backslash C} \sum_{k=K_{s t}(\mathcal{C})+1}^{K_{s t}} z_{s t}^{k} \leq|C|-\sum_{\sigma \in \mathcal{C}} x_{\sigma} \quad \forall t \in \mathcal{S} * \mathcal{T}, C \subseteq \mathcal{S}_{t}
$$

These inequalities have a similar structure as cover inequalities for a knapsack problem and can be viewed as GUB cover inequalities [52, 18]. Hence, we use the ambiguous term "cover" to denote $C$ in the present context. The complete model, which we call the discrete CQIs formulation and denote by the acronym DCF henceforth, then reads

$$
\begin{array}{ll}
\min \sum_{s \in \mathcal{S}} c_{s} x_{s}+\lambda \sum_{t \in \mathcal{T}} u_{t} & \\
\text { s.t. } \sum_{s \in \mathcal{S}_{t}} \sum_{k=1}^{\kappa_{s t}} z_{s t}^{k}+u_{t}=1 & \forall t \in \mathcal{T} \\
\sum_{t \in \mathcal{T}_{s}} \sum_{k=1}^{\kappa_{s t}} \frac{w_{t}}{e^{k}} z_{s t}^{k} \leq b_{s} x_{s} & \forall s \in \mathcal{S} \\
\sum_{s \in \mathcal{S}_{t} \backslash \mathcal{C}} \sum_{k=\kappa_{s t}(\mathcal{C})+1}^{\kappa_{s t}} z_{s t}^{k} \leq|\mathcal{C}|-\sum_{\sigma \in \mathcal{C}} x_{\sigma} & \forall t \in \mathcal{T}, C \subseteq \mathcal{S}_{t} \\
\sum_{k=1}^{K_{s t}} z_{s t}^{k} \leq x_{s} & \forall(s, t) \in \mathcal{S} * \mathcal{T} \\
x_{s}, z_{s t}^{k}, u_{t} \in\{0,1\}, &
\end{array}
$$

This ILP further represents the basic formulation (3) with adapted coverage (3b), capacity $3 \mathrm{c}$, and variable upper bound constraints $3 \mathrm{~d}$ ) in terms of the new variables and corresponding spectral efficiencies; see constraints (14b), 14c), and 14e). 
Lemma 2. The DCF (14) models the CNPP regarding interference via SINR conditions correctly, i.e.,

i) no SINR condition is violated by a feasible solution,

ii) no capacity is exceeded by a feasible solution.

Proof. By means of the cover constraints $[14 \mathrm{~d}]$, we prohibit the assignment of a TN $t$ to a BS $s$ with an insufficient link quality. Thus, for any feasible solution of the ILP (14), no violated SINR condition (4) can exist. Moreover, since we use the spectral efficiencies corresponding to the chosen CQI, no capacity violation can occur.

The ILP (14) can be computationally challenging since there might exist exponentially many constraints $14 \mathrm{~d}$ due to the existence of exponentially many covers $C$. Actually, (14) comprises at most $|\mathcal{S}|+|\mathcal{T}|+15 \cdot|\mathcal{S} * \mathcal{T}|$ variables and at most $|\mathcal{S}|+|\mathcal{T}|+$ $|\mathcal{S} * \mathcal{T}|+\sum_{t \in \mathcal{T}} 2^{\left|\mathcal{S}_{t}\right|}$ constraints. We present a way to deal with the formulation size by means of separation in the following paragraph.

Separation of Cover Inequalities. To decrease the size of DCF considerably, we neglect the cover inequalities (14d) and separate only violated inequalities on the fly for (fractional) solutions $(\tilde{x}, \tilde{z}, \tilde{u})$. We present an enumerative separation algorithm, which determines all possible covers $C$ and maximal CQIs $\kappa_{s t}(C)$ for each TN $t$ with $\tilde{u}_{t}<1$. If the corresponding inequality (14d) is violated, we add it to the formulation.

First, we define the set of (partially) deployed BSs as $\Psi:=\left\{s \in \mathcal{S} \mid \tilde{x}_{s}>0\right\}$. Based on $\Psi$, we define $\Psi_{t}:=\mathcal{S}_{t} \cap \Psi$ for all $t \in \mathcal{T}$ with $\tilde{u}_{t}<1$. We can then compute every subset $C \subseteq \Psi_{t}$ and the corresponding values $\kappa_{s t}(C)$ for all $s \in \mathcal{S}_{t} \backslash C$. If a violation of inequality 1 d4d identified, we have found a violated cover inequality, which we add to the cut pool. The complete routine is summarized in the algorithm stated in Fig 1 . briefly denoted as Algorithm 1 .

If this algorithm does not return a cover, then there does not exist a violated inequality (14d) for the current solution since all possible covers have been tested. Hence, the presented separation routine is exact.

For integer solutions $(\tilde{x}, \tilde{z}, \tilde{u})$, it is not efficient to run Algorithm 1 . Since the deployment of BSs and the TN assignment is fixed for a given solution, we simply 


\section{Algorithm 1}

Input: $\operatorname{solution}(\tilde{x}, \tilde{z}, \tilde{u})$

Output: $C$ and $\kappa_{s t}(C) \forall s \in \mathcal{S}_{t} \backslash C$ with violated inequality of type (14d) or proof that none exists

define $\Psi:=\left\{s \in \mathcal{S} \mid \tilde{x}_{s}>0\right\}$ and $\Psi_{t}:=\mathcal{S}_{t} \cap \Psi$ for all $t \in \mathcal{T}$ with $\tilde{u}_{t}<1$

for $t \in \mathcal{T}$ with $\tilde{u}_{t}<1$ do

for $C \subseteq \Psi_{t}$ do

for $s \in \Psi_{t} \backslash C$ with $\sum_{k=1}^{k_{s t}} \tilde{z}_{s t}^{k}>0$ do

compute SINR value $\gamma_{s t}(C):=\frac{P_{\mathrm{r}}(s, t)}{\sum_{\sigma \in \mathcal{C}} P_{\mathrm{r}}(\sigma, t)+\eta}$

define value for $\kappa_{s t}(C)$ based on $\gamma_{s t}(C)$ and Table 1

end for

end for

if $\sum_{s \in \Psi_{t} \mid C: \tilde{z}_{s t}>0} \sum_{k=\kappa_{s t}(\mathcal{C})+1}^{K_{s t}} \tilde{z}_{s t}^{k}>|C|-\sum_{\sigma \in C} \tilde{x}_{\sigma}$ then

compute SINR values $\gamma_{s t}(C)$ and corresponding $\kappa_{s t}(C) \forall s \in \mathcal{S}_{t} \backslash \Psi_{t}$ and $s \in \Psi_{t} \backslash C$

with $\sum_{k=1}^{K_{s t}} \tilde{z}_{s t}^{k}=0$

return $C$ and $\kappa_{s t}(C) \forall s \in \mathcal{S}_{t} \backslash C$

end if

end for

Figure 1: Enumerative separation of cover inequalities 


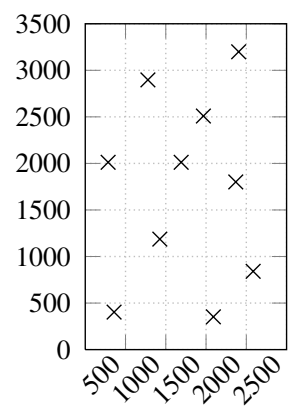

(a) set "a"

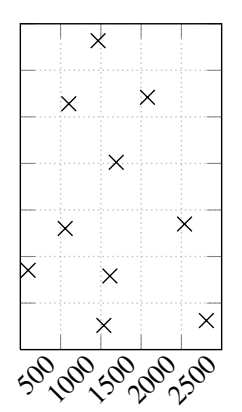

(b) set "b"

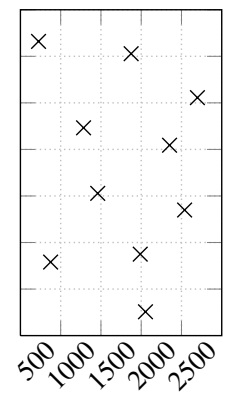

(c) set "c"

Figure 2: Three selected sets of $10 \mathrm{BSs}$ in a 2,500 $\times 3,500 \mathrm{~km}$ area of the used test scenarios.

compute the SINR value for every TN $\tilde{t}$ with serving BS $\tilde{s}$ and test if it is sufficiently large. If not, we add the cover inequality (14d) corresponding to TN $\tilde{t}$ and cover $C:=\left\{\sigma \in \mathcal{S}_{\tilde{t}} \mid \tilde{x}_{\sigma}=1\right\} \backslash\{\tilde{s}\}$. As is common practice, we separate cover inequalities for fractional solutions in the root node and for integer solutions in all nodes of the branch-and-cut algorithm.

\section{Numerical Evaluation}

In this section, a numerical evaluation of the five proposed formulations is presented. Before we compare them regarding SINR and capacity accuracy (Sections 5.2 and 5.3 respectively), we introduce the instances used. Afterwards, we compare the exact formulations w.r.t. computation times (Section 5.4.

\subsection{Scenarios and Settings}

The investigated test scenarios are based on signal propagation data for Munich, available at [53]. This data set comprises $60 \mathrm{BS}$ candidate sites from which we choose three sets of 10 BSs each, as displayed in Fig. 2. For simplicity, a BS candidate is limited to the location of the BS and we consider only path loss in the computation of the gain $a_{s t}$, i. e., $a_{s t}=10^{-\frac{1}{10} P_{\mathrm{L}}^{\mathrm{dB}}(s, t)}$ with $P_{\mathrm{L}}^{\mathrm{dB}}(s, t)$ denoting the path loss of the signal from $s$ to $t$ given in $\mathrm{dB}$. We use a cube oriented ray launching algorithm [54] for the computation of the path loss. Assuming a transmission power $p_{s}$ of $46 \mathrm{dBm}$ for every 
Table 2: Traffic profiles for TNs.

\begin{tabular}{c|cc}
\hline service & usage $(\%)$ & bit rate $(\mathrm{kbps})$ \\
\hline data & {$[10,20]$} & {$[512,2000]$} \\
web & {$[20,40]$} & {$[128,512]$} \\
VoIP & remaining & 64 \\
\hline
\end{tabular}

BS $s \in \mathcal{S}$, we compute the SNR value $\gamma=\frac{P_{\mathrm{r}}(s, t)}{\eta}$ for every $(s, t) \in \mathcal{S} * \mathcal{T}$ and extract the best possible spectral efficiency $\bar{e}_{s t}$ from Table 1. Additionally, we set $b_{s}=10 \mathrm{MHz}$ (cf. [41, Chapter 19]). The noise value in $\mathrm{dB}$ is computed as thermal noise (dB) plus noise figure for users (set to $9 \mathrm{~dB}$ ), where the temperature used in the thermal noise is set to $290 \mathrm{~K}$. Moreover, we define $c_{s}=4$ for every BS $s$, which represents the scaled value of $4000 \mathrm{~W}$ depicting the (rounded) total power consumed by a BS; cf. [55]. Based on the experiments in [36], the scaling parameter $\lambda$ is set to 1 .

For each BS set, we consider five different numbers of TNs, 100, 200, 300, 400 and 500, where the TNs are distributed randomly in the considered area. A test scenario is denoted by the number of TNs and the appended character of the BS set, e. g., "100a" denotes the combination of BS set "a" and 100 TNs.

Since real-life data of mobile users is not available due to data privacy limitations, we generate the demand values $w_{t}$ for each $t \in \mathcal{T}$ randomly from the user profiles in Table 2 (cf. [56] and Chapter 19 in [41]). A percentage for both data (downloading) and web (browsing) services is uniformly drawn from the "usage" column and multiplied by a bit rate uniformly drawn from the "bit rate" column. The remaining percentage is used for Voice-over-IP (VoIP) telephony with a bit rate of $64 \mathrm{kbps}$. The computed value is then rounded up to integer kbps. As an example, the minimum required bit rate of any $\mathrm{TN}$ is

$$
\lceil 10 \% \cdot 512 \mathrm{kbps}+20 \% \cdot 128 \mathrm{kbps}+70 \% \cdot 64 \mathrm{kbps}\rceil=122 \mathrm{kbps} .
$$

Furthermore, we define the SINR threshold as $\delta=-5.1 \mathrm{~dB}$, which is the lowest possible value to establish a link, and the corresponding minimum required spectral efficiency is $e_{\min }=0.25 \mathrm{bps} / \mathrm{Hz}$. For the TCRF we set the threshold $\delta_{\mathrm{c}}$ to 1.0 based 
Table 3: Number of TNs marked as uncovered in the solution and number of violated SINR requirements for CGF and TCRF.

\begin{tabular}{c|cccc}
\hline & \multicolumn{2}{|c}{ CGF } & \multicolumn{2}{c}{ TCRF } \\
scenario & \#uncov & \#SINR & \#uncov & \#SINR \\
\hline $100 \mathrm{a}$ & 2 & 4 & 2 & 0 \\
$100 \mathrm{~b}$ & 0 & 15 & 0 & 1 \\
$100 \mathrm{c}$ & 1 & 15 & 2 & 1 \\
$200 \mathrm{a}$ & 0 & 0 & 0 & 0 \\
$200 \mathrm{~b}$ & 0 & 9 & 0 & 3 \\
$200 \mathrm{c}$ & 3 & 15 & 0 & 7 \\
$300 \mathrm{a}$ & 0 & 29 & 0 & 0 \\
$300 \mathrm{~b}$ & 2 & 2 & 0 & 4 \\
$300 \mathrm{c}$ & 1 & 9 & 1 & 2 \\
$400 \mathrm{a}$ & 0 & 10 & 0 & 1 \\
$400 \mathrm{~b}$ & 0 & 26 & 0 & 6 \\
$400 \mathrm{c}$ & 0 & 33 & 0 & 16 \\
$500 \mathrm{a}$ & 0 & 39 & 2 & 10 \\
$500 \mathrm{~b}$ & 1 & 23 & 1 & 8 \\
$500 \mathrm{c}$ & 0 & 36 & 0 & 49 \\
\hline
\end{tabular}

on the numerical study performed in [3]. For better comparability, discrete spectral efficiencies are derived according to Table 1 for TOF.

All computations are performed on a Linux machine with $3.40 \mathrm{GHz}$ Intel Core i73770 processor, 32 GB RAM, and a general CPU time limit of twelve hours. Additionally, we limit the available RAM to 31 GB (to leave some for the system) and use ILOG CPLEX 12.6 [57] as (I)LP solver.

\subsection{Evaluation of SINR Approximation}

In our first numerical evaluation, we compare the two approaches which approximate the SINR constraints; the CGF and the TCRF. From the optimal solutions obtained by these two formulations, we compute the correct SINR values according to (1) and count the number of violated SINR conditions. In Table 3 , we depict the number of uncovered TNs $\left(u_{t}=1\right)$ in the optimal solutions and the number of violated SINR requirements. The numbers of TNs marked as covered are quite similar for both ap- 
Table 4: Number of deployed BSs and covered TNs and the maximum load for SCF, TCRF, TOF, and DCF.

\begin{tabular}{|c|c|c|c|c|c|c|c|c|c|c|c|c|}
\hline \multirow[b]{2}{*}{ scenario } & \multicolumn{3}{|c|}{$\mathrm{SCF}$} & \multicolumn{3}{|c|}{ TCRF } & \multicolumn{3}{|c|}{ TOF } & \multicolumn{3}{|c|}{$\mathrm{DCF}$} \\
\hline & BSs & TNs & max. load & BSs & TNs & max. load & BSs & TNs & max. load & BSs & TNs & $\max$. load \\
\hline $100 \mathrm{a}$ & 2 & 98 & 1.5 & 2 & 98 & 1.4 & 3 & 100 & 1.0 & 3 & 100 & 1.0 \\
\hline $100 \mathrm{~b}$ & 3 & 100 & 1.1 & 3 & 100 & 1.1 & 3 & 100 & 1.0 & 3 & 100 & 1.0 \\
\hline $100 \mathrm{c}$ & 2 & 97 & 1.2 & 2 & 98 & 1.3 & 3 & 100 & 0.9 & 3 & 100 & 0.9 \\
\hline $200 \mathrm{a}$ & 3 & 200 & 2.1 & 3 & 200 & 2.2 & 6 & 200 & 1.0 & 6 & 200 & 1.0 \\
\hline $200 b$ & 4 & 199 & 1.8 & 4 & 200 & 1.7 & 5 & 194 & 1.0 & 6 & 198 & 1.0 \\
\hline $200 \mathrm{c}$ & 4 & 200 & 1.6 & 4 & 200 & 2.0 & 6 & 200 & 1.0 & 6 & 200 & 1.0 \\
\hline $300 \mathrm{a}$ & 4 & 300 & 3.1 & 4 & 300 & 2.5 & 7 & 284 & 1.0 & 7 & 284 & 1.0 \\
\hline $300 \mathrm{~b}$ & 4 & 298 & 2.9 & 5 & 300 & 2.2 & 9 & 297 & 1.0 & 9 & 297 & 1.0 \\
\hline $300 \mathrm{c}$ & 4 & 299 & 4.0 & 4 & 299 & 2.4 & 9 & 294 & 1.0 & 9 & 294 & 1.0 \\
\hline $400 \mathrm{a}$ & 4 & 399 & 4.0 & 5 & 400 & 2.7 & 10 & 377 & 1.0 & $10^{*}$ & $377 *$ & $1.0 *$ \\
\hline $400 \mathrm{~b}$ & 6 & 399 & 2.8 & 6 & 400 & 2.7 & 9 & 364 & 1.0 & 9 & 364 & 1.0 \\
\hline $400 \mathrm{c}$ & 5 & 400 & 3.4 & 5 & 400 & 3.4 & 9 & 364 & 1.0 & 9 & 364 & 1.0 \\
\hline $500 \mathrm{a}$ & 5 & 498 & 4.3 & 5 & 498 & 4.0 & 10 & 431 & 1.0 & $10^{*}$ & $431^{*}$ & $1.0 *$ \\
\hline $500 \mathrm{~b}$ & 6 & 494 & 3.9 & 7 & 499 & 2.9 & 9 & 422 & 1.0 & $9 *$ & $422 *$ & $1.0 *$ \\
\hline $500 \mathrm{c}$ & 6 & 499 & 4.1 & 6 & 500 & 3.7 & 10 & 412 & 1.0 & $10^{*}$ & $412 *$ & $1.0 *$ \\
\hline
\end{tabular}

proaches. Moreover, a tendency for higher numbers of violated SINR requirements when the number of TNs increases is apparent for both formulations. As claimed in Section 3.2, the conflict graph actually limits the number of SINR violations. However, the resulting effect is not satisfactory as up to $15 \%$ of the TNs are essentially not covered due to an insufficient SINR. Furthermore, except for scenarios 300b and 500c, the TCRF violates significantly less SINR conditions (only up to $4 \%$ ) than the CGF. Hence, the latter formulation approximates the SINR conditions better than the conflict graph which we do not consider further.

\subsection{Evaluation of Capacity Approximation}

We now analyze the quality of the capacity approximation for the SCF as well as the TCRF in comparison with the exact solutions obtained from the TOF and the DCF. To this end given a solution, we first define the load of a BS $s \in \mathcal{S}^{\prime}$, where $\mathcal{S}^{\prime}$ denotes the set of deployed BSs, i. e., $\mathcal{S}^{\prime}=\left\{s \in \mathcal{S} \mid x_{s}=1\right\}$. Denoting by $\mathcal{T}_{s}^{\prime}$ the set of TNs 


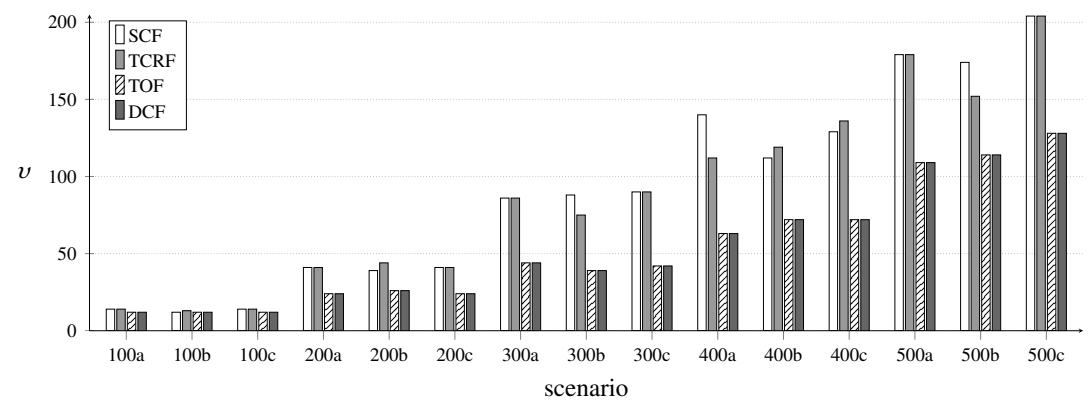

Figure 3: Corrected objective values $v$ for all scenarios and investigated formulations (for $c_{s}=4$ and $\lambda=1$ ).

assigned to $s$, the load (as percentage) is given as

$$
\ell_{s}:=\frac{1}{b_{s}} \sum_{t \in \mathcal{T}_{s}^{\prime}} \frac{w_{t}}{e_{s t}^{\prime}},
$$

with $e_{s t}^{\prime}$ denoting the spectral efficiency corresponding to the actual SINR value

$$
\frac{P_{\mathrm{r}}(s, t)}{\sum_{\sigma \in I_{s t}} P_{\mathrm{r}}(\sigma, t)+\eta} .
$$

The set $I_{s t} \subset \mathcal{S}^{\prime}$ comprises the interfering and deployed BSs of the signal from $s$ to $t$. Note that $\ell_{s}>1$ indicates a capacity violation.

Table 4 shows the number of deployed BSs (out of 10), the number of TNs regarded as covered, and the maximum load for all four considered formulations SCF, TCRF, TOF, and DCF. The numbers marked by a * for DCF are not labeled as optimal by the solver since the amount of memory is insufficient for the solution process; the largest optimality gap is $2.8 \%$. However, we can deduce from the solutions obtained by TOF that the solutions for scenarios 400a and 500a-c obtained by DCF are indeed optimal.

The numbers for the two exact approaches are usually identical as expected, except for scenario 200b, where the two distinct solutions give the same objective value. Apart from scenario 100b, the SCF as well as the TCRF deploys notably less BSs than the exact solution since these approaches do not model the capacity constraints exactly. In the exact solution, the accurate modeling of interference and bandwidth consumption has a twofold implication: more BSs are necessary to satisfy the demand of the covered TNs and (nonetheless) more TNs are left uncovered. In general, in the SCF as well as in 
Table 5: Solving times (in s) of the TOF, the DCF, and of Algorithm 1 for scenarios solved to optimality.

\begin{tabular}{l|cccccccccccc}
\hline scenario & $100 \mathrm{a}$ & $100 \mathrm{~b}$ & $100 \mathrm{c}$ & $200 \mathrm{a}$ & $200 \mathrm{~b}$ & $200 \mathrm{c}$ & $300 \mathrm{a}$ & $300 \mathrm{~b}$ & $300 \mathrm{c}$ & $400 \mathrm{a}$ & $400 \mathrm{~b}$ & $400 \mathrm{c}$ \\
\hline TOF & 6.7 & 3.4 & 10.2 & 110.1 & 14.2 & 35.7 & 1035.7 & 96.6 & 532.3 & 18190.1 & 136.5 & 628.1 \\
DCF & 4.7 & 2.5 & 9.8 & 39.1 & 14.6 & 36.0 & 1654.1 & 46.8 & 262.8 & memory & 576.2 & 3511.0 \\
Alg. 1 1 & 3.7 & 1.2 & 15.5 & 2035.3 & 105.8 & 388.2 & 3600.4 & 955.9 & 9204.1 & time & 2036.0 & time \\
\hline
\end{tabular}

the TCRF more TNs are assigned (due to fewer interfering BSs), but such assignments cause serious overload situations (after recalculating the bandwidth consumption) as indicated by the columns "max. load" and many TNs will be dropped in practice. As an example, a value of 4.0 indicates that one BS receives the load that should be handled by four BSs. Hence, both approximate formulations violate the capacity constraints severely.

To demonstrate this violation more clearly, we perform the following postprocessing step for every approximate solution. For a set $\mathcal{S}^{\prime}$ of deployed BSs, we solve the TN assignment problem

$$
\begin{array}{rlr}
\tau:=\min & \sum_{t \in \mathcal{T}} u_{t} \\
\text { s.t. } & \sum_{s \in \mathcal{S}^{\prime}} z_{s t}+u_{t}=1 & \forall t \in \mathcal{T} \\
& \sum_{t \in \mathcal{T}_{s}} \frac{w_{t}}{e_{s t}^{\prime}} z_{s t} \leq b_{s} & \forall s \in \mathcal{S}^{\prime} \\
& u_{t}, z_{s t} \in\{0,1\} &
\end{array}
$$

with the correct spectral efficiencies $e_{s t}^{\prime}$. By ILP (15), we count the true number of unassigned TNs for a given set of deployed BSs when allowing a complete new assignment. We denote this number (the objective value) by $\tau$. Then, we define the corrected objective value $v$ as follows.

$$
v:=\sum_{s \in \mathcal{S}^{\prime}} c_{s}+\lambda \tau
$$

If a solution does not violate any capacity constraint nor SINR requirement, $v$ is equal to the objective value (3a). This is the case for TOF as well as DCF.

In Fig. 3, we illustrate $v$ for SCF, TCRF, TOF, and DCF. For the three smallest 


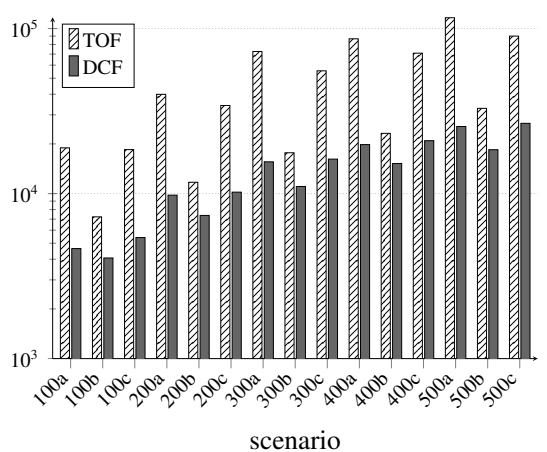

(a) variables

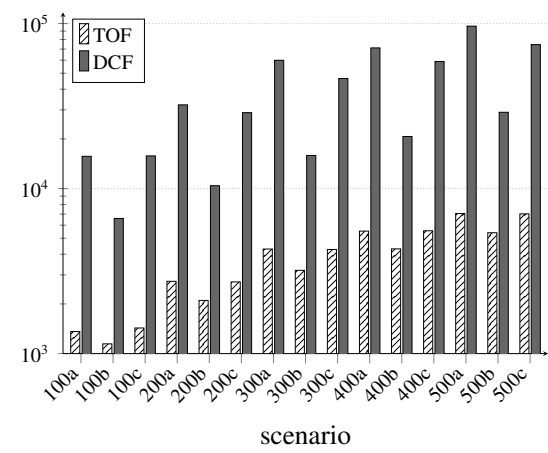

(b) constraints

Figure 4: Number of variables and constraints for the TOF and DCF and all scenarios.

scenarios comprising $100 \mathrm{TNs}$, the values of $v$ for the four formulations are at close quarters. However, for an increasing number of TNs, the corrected objective values of the approximate formulations become noticeably larger than the exact values due to serious capacity violations. Comparing the two approximate formulations, it is not possible to judge which formulation violates the capacity constraints more severely in general. For the SCF, the percentage increase of the corrected objective value compared to the exact value ranges from 0 to $126 \%$ where $0 \%$, i. e., the optimal objective value, is obtained by reassigning the TNs for scenario 100b. The percentage increase of the corrected objective value lies between 8 and $114 \%$ for the TCRF.

\subsection{Comparison of the Exact Formulations}

Now, let us compare the exact formulations on their pros and cons. First, we compare the number of variables and constraints per scenario for both exact formulations in Fig. 4 (note the logarithmic scale). By the definition of the ILPs (12) and (14), the TOF comprises significantly more variables than the DCF for which it is the other way round for the number of constraints. However, the number of constraints for the TOF is always lower than the number of variables for the other formulation. Especially for the scenarios with $500 \mathrm{TNs}$ this observation becomes more important as the TOF solves these scenarios while the DCF exceeds the memory limit for all three scenarios. Thus, for the CNPP with interference modeling, a high number of constraints is 
Table 6: Number of installed BSs and covered TNs, optimality gap in \% and solving time in s for DCF combined with the separation algorithm 1 and scenarios with 28 BSs.

\begin{tabular}{l|rrrr}
\hline scenario & \# BSs & \# TNs & gap (\%) & time (s) \\
\hline $28 \_100$ & 2 & 99 & 0.0 & 331 \\
$28 \_200$ & 5 & 200 & 0.0 & 41076 \\
$28 \_300$ & 8 & 299 & 68.2 & 43200 \\
$28 \_400$ & 0 & 0 & 2138.4 & 43200 \\
\hline
\end{tabular}

computationally more difficult than a high number of variables.

In Table 5, we present the solving times for both exact formulations, including Algorithm 1 1 which we will discuss later, and all scenarios with up to 400 TNs. For the scenarios with 100 TNs and for scenario 200a, the DCF is faster. But for an increasing number of TNs, this trend reverses in general as the number of constraints becomes more substantial. The TOF in particular performs much better for scenario 400a. Thus, from the presented results we could conclude that TOF should be favored over DCF. However, the performance of the former approach depends considerably more on the number of candidate BSs as demonstrated in the following.

We construct a new set of BSs by consolidating sets "a", "b", and "c" resulting in a set of 28 BSs (two BS occur twice). Following the description in Section 5.1, we create scenarios with 100, 200, 300 and 400 TNs. Though, none of these scenarios can be solved by TOF nor DCF due to excessive memory usage. But, as described in the paragraph on the separation of cover inequalities in Section 4.2, we can decrease the size of DCF (14) considerably. By means of the separation Algorithm 1 , we can determine (optimal) solutions for (some) scenarios. To apply the separator, we set the frequency to 1, i. e., Algorithm 1 is called at every node of the branch-and-bound tree. Other frequency choices such as 0 (call only at root node) were outperformed by the frequency 1 in preliminary tests; see [3].

For the scenarios comprising 28 BSs (denoted by “28_\#TNs"), we present in Table 6 the number of deployed BSs and covered TNs, the optimality gap and the solving time achieved by the DCF combined with the separation algorithm 1]. We observe a 
degradation in performance for an increasing number of TNs. Table 5 shows the same effect for scenarios comprising $10 \mathrm{BSs}$.

The final presented results for DCF clearly demonstrate the advantage of the DCF over the TOF in case of a large number of BS candidates since we did not obtain any solution for such test scenarios with TOF.

Although the computations show that the newly proposed models are computationally tractable for small network sizes, the models will be too demanding for the optimization of larger networks. The accurate modeling of SINR and bandwidth consumption is merely to be used in scenarios of network expansion, network changes, resolution of local quality of service issues, and local verification of heuristically developed larger network plans. In such case, only a few BSs are involved and exact results can be achieved this way.

\section{Conclusions}

In this paper, we have investigated interference modeling in cellular networks. To this end, we have presented three established approaches which are based on either SINR conditions via cover inequalities or (conflict) graphs. Though remarkably fast, all these formulations violate capacity constraints and the graph-based models violate also SINR constraints. Therefore, we have developed two novel formulations; on the one hand, a set-wise model, the TN oriented formulation and, on the other hand, a model based on discrete values for the quality of a link, the discrete CQIs formulation. Both formulations are exact in terms of SINR requirements as well as capacity constraints but also comprise an exponential number of either variables or constraints.

A numerical evaluation performed on fifteen scenarios comprising ten BSs and 100 to $500 \mathrm{TNs}$ has revealed the insufficiency of the conflict graph formulation regarding the modeling of SINR conditions. Furthermore, the two other approximate formulations suffer from severe capacity violations as demonstrated by a postprocessing step which solves the TN assignment problem for a predefined set of installed BSs.

A comparison of the two exact approaches has revealed the predominance of the TN oriented formulation for a small number of BS candidates. Further, for a signif- 
icantly larger number of BSs, the working size of the discrete CQIs formulation can be reduced by means of a separation algorithm such that this formulation is the only model being able to solve the larger test scenarios. The improvement of the separation routine remains as future work to solve scenarios with more than 200 TNs (optimally). Similarly, improving the performance of the TN-oriented formulation remains as future work. This can be done by implementing a branch-and-price framework where variables are generated on the fly. Finally, as the proposed approaches do not scale well with the number of BSs (and TNs) considered, ways to incorporate the results in systems to optimize larger networks should be considered, e.g., by round-robin approaches or local search.

\section{Acknowledgment}

This work was supported by the DFG research grant KO2311/3-1, SCHM2643/5-1 as well as European H2020 MSCA project DECADE (grant number: 645705). The

authors would like to thank two anonymous referees for their valueable comments to improve the paper.

\section{References}

[1] K. Aardal, S. van Hoesel, A. M. C. A. Koster, C. Mannino, A. Sassano, Models and solution techniques for frequency assignment problems, Annals of Operations Research 153 (2007) 79-129.

[2] P. Gupta, P. Kumar, The capacity of wireless networks, IEEE Transactions on Information Theory 46 (2000) 388-404.

[3] G. Claßen, Optimisation under Data Uncertainty in Wireless Communication Networks, Ph.D. thesis, RWTH Aachen University, Aachen (2015).

URL https://publications.rwth-aachen.de/record/465292

[4] E. Amaldi, A. Capone, F. Malucelli, C. Mannino, Optimization problems and models for planning cellular networks problems, in: M. Resende, P. Pardalos 
(Eds.), Handbook of Optimization in Telecommunication, Springer, 2006, pp. 917-939.

[5] J. Kennington, E. Olinick, D. Rajan (Eds.), Wireless Network Design: Optimization Models and Solution Procedures, Springer, 2006.

[6] T. Moscibroda, R. Wattenhofer, The Complexity of Connectivity in Wireless Networks, in: 25th IEEE International Conference on Computer Communications (INFOCOM 2006), 2006, pp. 1-13.

[7] M. Andrews, M. Dinitz, Maximizing Capacity in Arbitrary Wireless Networks in the SINR Model: Complexity and Game Theory, in: IEEE INFOCOM, 2009, pp. $1332-1340$.

[8] Y. Li, M. Pióro, D. Yuan, J. Su, Optimizing compatible sets in wireless networks through integer programming, EURO Journal on Computational Optimization 2 (2014) 1-15.

[9] V. Ramamurthi, A. Reaz, D. Ghosal, S. Dixit, B. Mukherjee, Channel, capacity, and flow assignment in wireless mesh networks, Computer Networks 55 (2011) $2241-2258$.

[10] A. Eisenblätter, R. Wessäly, A. Martin, A. Fügenschuh, O. Wegel, T. Koch, T. Achterberg, A. M. C. A. Koster, Modelling Feasible Network Configurations for UMTS, in: G. Anandalingam, S. Raghavan (Eds.), Telecommunications Network Design and Management, Vol. 23 of Operations Research/Computer Science Interfaces Series, Springer US, 2003, pp. 1-23.

[11] A. Eisenblätter, H. Geerdes, T. Koch, A. Martin, R. Wessäly, UMTS radio network evaluation and optimization beyond snapshots, Mathematical Methods of Operations Research 63 (2006) 1-29.

[12] E. Amaldi, A. Capone, F. Malucelli, Radio Planning and Coverage Optimization of 3G Cellular Networks, Wireless Networks 14 (2008) 435-447. 
[13] F. D'Andreagiovanni, On Improving the Capacity of Solving Large-scale Wireless Network Design Problems by Genetic Algorithms, in: Proceedings of European Conference on the Applications of Evolutionary Computation, Springer Lecture Note on Computer Science vol. 6625, 2011, pp. 11-20.

[14] F. D'Andreagiovanni, G. Gleixner, Towards an Accurate Solution of Wireless Network Design Problems, in: Proceedings of the 4th International Symposium on Combinatorial Optimization, Springer Lecture Note on Computer Science vol. 9849, 2016, pp. 135-147.

[15] C. Mannino, F. Rossi, S. Smriglio, The Network Packing Problem in Terrestrial Broadcasting, Operations Research 54 (2006) 611-626.

[16] A. Capone, L. Chen, S. Gualandi, D. Yuan, A New Computational Approach for Maximum Link Activation in Wireless Networks under the SINR Model, IEEE Transactions on Wireless Communications 10 (2011) 1368-1372.

[17] F. D’Andreagiovanni, C. Mannino, A. Sassano, Negative Cycle Separation in Wireless Network Design, in: Proceedings of the International Network Optimization Conference, Springer Lecture Note on Computer Science vol. 6701, 2011, pp. 51-56.

[18] F. D’Andreagiovanni, C. Mannino, A. Sassano, GUB Covers and Power-Indexed Formulations for Wireless Network Design, Management Science 59 (2013) 142_156.

[19] F. D'Andreagiovanni, Pure 0-1 Programming Approaches to Wireless Network Design, Ph.D. thesis, Sapienza Universitá di Roma, Rome (2010).

[20] F. D'Andreagiovanni, Pure 0-1 programming approaches to wireless network design, 4OR 10 (2) (2012) 211-212. URL https://doi.org/10.1007/s10288-011-0162-z

[21] A. Eisenblätter, H. Geerdes, Capacity Optimization for UMTS: Bounds and Benchmarks for Interference Reduction, in: Proceedings of IEEE International 
Symposium on Personal, Indoor and Mobile Radio Communications, 2008, pp. $1-6$.

[22] D. Yuan, V. Angelakis, L. Chen, E. Karipidis, E. G. Larsson, On optimal link activation with interference cancellation in wireless networking, IEEE Transactions on Vehicular Technology 62 (2013) 939-945.

[23] B. Gendron, M. G. Scutellá, R. G. Garroppo, G. Nencioni, L. Tavanti, A Branchand-Benders-Cut Method for Nonlinear Power Design in Green Wireless Local area Networks, European Journal of Operational Research 255 (2016) 151-162.

[24] J. Naoum-Sawaya, S. Elhedhli, A Nested Benders Decomposition Approach for Optimal W-CDMA Telecommunication Network Planning, Naval Research Logistics 57 (2010) 151-162.

[25] F. Gu, , H. Liu, Y. Cheung, S. Xie, Optimal WCDMA Network Planning by Multiobjective Evolutionary Algorithm with Problem-specific Genetic Operation, Knowledge and Information Systems 45 (2011) 679-703.

[26] G. Koutitas, Green Network Planning of Single Frequency Networks, IEEE Transactions on Broadcasting 56 (2010) 541-550.

[27] A. Zakrzewska, F. D’Andreagiovanni, S. Ruepp, M. Berger, Biobjective Optimization of Radio Access Technology Selection and Resource Allocation in Heterogeneous Wireless Networks, in: Proceedings of WiOpt RAWNET Workshop, 2013, pp. 1-7.

[28] C. Büsing, F. D’Andreagiovanni, New Results about Multi-band Uncertainty in Robust Optimization, in: Proceedings of International Symposium on Experimental Algorithms, Springer Lecture Note on Computer Science vol. 7276, 2012, pp. 63-74.

[29] F. D’Andreagiovanni, Revisiting Wireless Network Jamming by SIR-based Considerations and Multiband Robust Optimization, Optimization Letters 9 (2015) $1495-1510$. 
[30] R. Garroppo, G. Nencioni, M. G. Scutellá, L. Tavanti, Robust Optimisation of Green Wireless LANs under Rate Uncertainty and User Mobility, Electronic Notes in Discrete Mathematics 52 (2016) 221-228.

[31] T. Heikkinen, A. Prékopa, Optimal Power Control in a Wireless Network Using a Model with Stochastic Link Coefficients, Naval Research Logistics 52 (2004) 178-192.

[32] E. Olinick, J. Rosenberger, Optimizing Revenue in CDMA Networks under Demand Uncertainty, European Journal of Operational Research 186 (2008) 812825.

[33] Atoll, Atoll Overview (2017). URL http://www. forsk.com/atoll/

[34] Ranplan, iBuildNet Professional (2017). URL https://www.ranplan.co.uk/products/

[35] A. Engels, M. Reyer, R. Mathar, Profit-Oriented Combination of Multiple Objectives for Planning and Configuration of 4G Multi-Hop Relay Networks, in: 7th Int. Symp. on Wireless Communication Systems (IEEE ISWCS), 2010, pp. $330-334$.

[36] G. Claßen, A. M. C. A. Koster, A. Schmeink, A Robust Optimisation Model and Cutting Planes for the Planning of Energy-Efficient Wireless Networks, Computers and Operations Research 40 (2013) 80-90.

[37] K. Tutschku, N. Gerlich, P. Tran-Gia, An Integrated Approach To Cellular Network Planning, in: Proceedings of the 7th International Network Planning Symposium Networks, 1996, pp. 185-190.

[38] S. Sesia, I. Toufik, M. Baker, LTE, The UMTS Long Term Evolution: From Theory to Practice, Wiley, 2009.

[39] L. A. Wolsey, Integer programming, Wiley-Interscience, New York, NY, USA, 1998. 
[40] J. Grönkvist, A. Hansson, Comparison Between Graph-based and Interferencebased STDMA Scheduling, in: Proceedings of the 2nd ACM International Symposium on Mobile Ad Hoc Networking \& Computing (MobiHoc), ACM, New York, NY, USA, 2001, pp. 255-258.

[41] F. Khan, LTE for 4G Mobile Broadband, Cambridge University Press, 2009.

[42] R. Mathar, T. Niessen, Optimum positioning of base stations for cellular radio networks, Wireless Networks 6 (2000) 421-428.

[43] J. Riihijarvi, M. Petrova, P. Mahonen, Frequency allocation for WLANs using graph colouring techniques, in: Second Annual Conference on Wireless Ondemand Network Systems and Services (WONS), 2005, pp. 216-222.

[44] A. Engels, M. Neunerdt, R. Mathar, H. Abdullah, Acceptance as a Success Factor for Planning Wireless Network Infrastructure, in: International Symposium on Wireless Communication Systems (ISWCS), Aachen, Germany, 2011, pp. 889893.

[45] Z. Niu, S. Zhou, Y. Hua, Q. Zhang, D. Cao, Energy-Aware Network Planning for Wireless Cellular System with Inter-Cell Cooperation, IEEE Transactions on Wireless Communications 11 (2012) 1412-1423.

[46] M. Padberg, On the Facial Structure of Set Packing Polyhedra, Mathematical Programming 5 (1973) 199-215.

[47] R. M. Karp, Reducibility among combinatorial problems, in: R. E. Miller, J. W. Thatcher (Eds.), Complexity of Computer Computations, Plenum, 1972, pp. 85103.

[48] C. Bron, J. Kerbosch, Algorithm 457: finding all cliques of an undirected graph, Communications of the ACM 16 (1973) 575-577.

[49] E. Tomita, A. Tanaka, H. Takahashi, The worst-case time complexity for generating all maximal cliques and computational experiments, Theoretical Computer Science 363 (2006) 28-42. 
[50] M. C. Schmidt, N. F. Samatova, K. Thomas, B.-H. Park, A scalable, parallel algorithm for maximal clique enumeration, Journal of Parallel and Distributed Computing 69 (4) (2009) $417-428$.

[51] A. Engels, M. Reyer, X. Xu, R. Mathar, J. Zhang, H. Zhuang, Autonomous SelfOptimization of Coverage and Capacity in LTE Cellular Networks, IEEE Transactions on Vehicular Technology 62 (2013) 1989-2004.

[52] L. A. Wolsey, Valid inequalities for 0-1 knapsacks and MIPs with generalised upper bound constraints, Discrete Applied Mathematics 29 (1990) 251-261.

[53] COST 231, Urban micro cell measurements and building data, accessed 30.08.2014 (1996).

URL http://www2.ihe.uni-karlsruhe.de/forschung/cost231/ cost231.en.html

[54] R. Mathar, M. Reyer, M. Schmeink, A Cube Oriented Ray Launching Algorithm for 3D Urban Field Strength Prediction, in: IEEE International Conference on Communications (ICC), 2007, pp. 5034-5039.

[55] M. Deruyck, W. Vereecken, E. Tanghe, W. Joseph, M. Pickavet, L. Martens, P. Demeester, Comparison of power consumption of mobile WiMAX, HSPA and LTE access networks, in: 9th Conference on Telecommunications Internet and Media Techno Economics (CTTE), 2010, pp. 1-7.

[56] A. Engels, Dimensioning, cell site planning, and self-organization of $4 \mathrm{~g}$ radio networks, Ph.D. thesis, RWTH Aachen University (2013).

[57] IBM - ILOG, CPLEX Optimization Studio 12.6 (2013). URL http://www.ilog.com/products/cplex 